\title{
AMYL NITRITE TEST IN PRIMARY GLAUCOMA*
}

BY

\author{
G. CRISTINI and N. PAGLIARANI \\ Clinica Oculistica Universitaria di Bologna. Direttore: Prof. Q. Di Marzio
}

THOUGH ocular hypertension is one of the fundamental symptoms of glaucoma, we have as yet little clinical evidence for an evaluation of its pathological significance and real severity. The only elements at our disposal are those inferred by the study of the pressure curve both spontaneous and after miotic drugs. These studies show that at the onset of the disease the basic pressure may still be normal, in spite of a certain degree of instability; during the course of the disease the tension rises with diurnal phasic variations of wide range; in the late stages of the disease these diurnal variations become smaller and the basic pressure rises to a very high level.

Leydhecker (1952) recently employed the usual provocative tests (waterdrinking, lability, mydriasis) to define the changes responsible for this symptom and evaluate its severity. His attempts led to uncertain conclusions, because our knowledge of the function primarily impaired, and of the exact mechanism of the tests employed is uncertain.

In fact, the possibility of solving this problem depends upon the exact knowledge of the pathogenesis of hypertension as well as of the mechanism of action of the tests employed.

With regard to the pathogenesis of the symptom, our views are confined to-day to two hypotheses which may be considered complementary. The first, elaborating the old conception of Priestley Smith $(1879,1891)$, maintains that hypertension is caused by an anatomical obstacle to the outflow of the aqueous. This obstacle is to be sought, according to Ascher (1949), in the aqueous veins, and, according to Goldmann (1948), in the trabeculae of the anterior chamber. An anatomical obliteration in the anterior aqueous drainage channels would, however, lead to a blocking of circulation, which makes it hard to explain the clinical behaviour of intra-ocular pressure. A mechanical obstacle present at a certain hour of the day cannot possibly be absent at another hour of the same day, when tension falls (DukeElder, 1952). Thomassen (1948) suggests a functional hindrance to the aqueous outflow caused by increased pressure in the episcleral venous vessels, into which the aqueous flows. A second hypothesis put forward by one of us (Cristini, 1951a) postulates the vascular nature of hypertension. It would result from a rise in the hydrostatic pressures in the uveal capillary-

*Received for publication July $6,1953$. 
venous system in consequence of a progressive obliteration of the capillary network, by analogy with what is now generally accepted as the pathogenesis of the neural symptoms in glaucoma.

These two hypotheses do not exclude each other, but, as Urrets Zavalia (1952) asserts, they may be complementary: with the rise in capillary venous pressure the functional hindrance to the outflow of the aqueous would also occur simultaneously in the recipient episcleral venous vessels. In any case, the function primarily impaired would be the uveal vascular function.

In order to investigate the impairment of the functions assumed to be responsible for ocular hypertension, it appeared indispensable to employ a drug with a vasodilatory action on the small uveal vessels. We chose amyl nitrite because it is easy to use and rapid in action, and has a vasodilatory effect on the small vessels. These latter properties exclude any other mechanism which might otherwise influence the variations in pressure. The effects of this drug on intra-ocular pressure in glaucomatous patients had already been studied by Bailliart and Bollack (1921), who had observed, in three cases, a rise in ocular tension and a fall in brachial arterial pressure. Cristini (1947), in a larger number of glaucomatous patients, saw a fall in ocular tension which varied in each individual.

Assuming that hypertension in glaucoma is due to a pathological process of this nature, the effect of amyl nitrite on the small vessels may supply interesting information regarding the pathological conditions of the uveal circulation in glaucomatous patients, and the possibility of a widening of the capillary network in consequence of a dilatation of the lumina and the opening up of areas of vascular reserve.

\section{Technique}

The brachial pressure and ocular tension (Schiötz tonometer) were measured simultaneously; the patient was then made to inspire some drops of amyl nitrite. The brachial systolic pressure and ocular tension were repeatedly measured, first at short intervals of 10 to $15 \mathrm{sec}$., and then at intervals of about $30 \mathrm{sec}$. for a period of 4 to $5 \mathrm{~min}$.

To obtain fuller details of the conditions of uveal circulation, the research was carried out in three conditions:

(1) in basic conditions, i.e., without previous instillation of drugs, to find out to what degree the network of the small uveal vessels was able to widen in relation to the different stages of glaucoma;

(2) after the instillation of cholinergic drugs which produce a dilatation of the small vessels and the opening up of new vascular areas (Cristini, 1947, 1949, 1951a, b; Duke-Elder, 1952; Urrets Zavalia, 1952);

(3) after blocking the stellate ganglion by means of novocain to abolish the sympathetic control, which acts chiefly on the small uveal vessels (Greaves and Perkins, 1952), with the purpose of examining the neural control of the widening of the capillary network.

The research was carried out on fifty glaucomatous eyes whose diurnal phasic variations had been previously studied before and after the instillation of cholin- 
ergic drugs (pilocarpine and eserine); the test was repeated several times in order to confirm the results and to find out if the diurnal phasic variations had any influence on them.

\section{Results}

The rapidity with which the changes in intra-ocular pressure appear after inspiration of amyl nitrite demonstrates the action of this drug on the small uveal vessels. The absence of relationship between the fall in brachial pressure and the fall in ocular tension, and the fact that the former very often followed the latter, permitted us to exclude a dependence of the uveal circulatory changes upon the general arterial pressure changes.

(1) In Basic Conditions.-The fall in intra-ocular pressure was larger in subjects who had already spontaneously shown extensive diurnal phasic variations and in those patients in whom the action of miotics had been more pronounced.

The fall was very small or absent in patients showing normal ocular tension, or during the absolute stage. In congestive glaucoma no fall in tension was observed; on the contrary, during the second phase of amyl nitrite inspiration a short rise in ocular tension above the initial values occurred (Fig. 1A).

(2) After Instillation of Cholinergic Drugs.-The fall in intra-ocular pressure was very small and was almost completely absent in those cases in which ocular tension had been normalized by the drug (Fig. 1B).

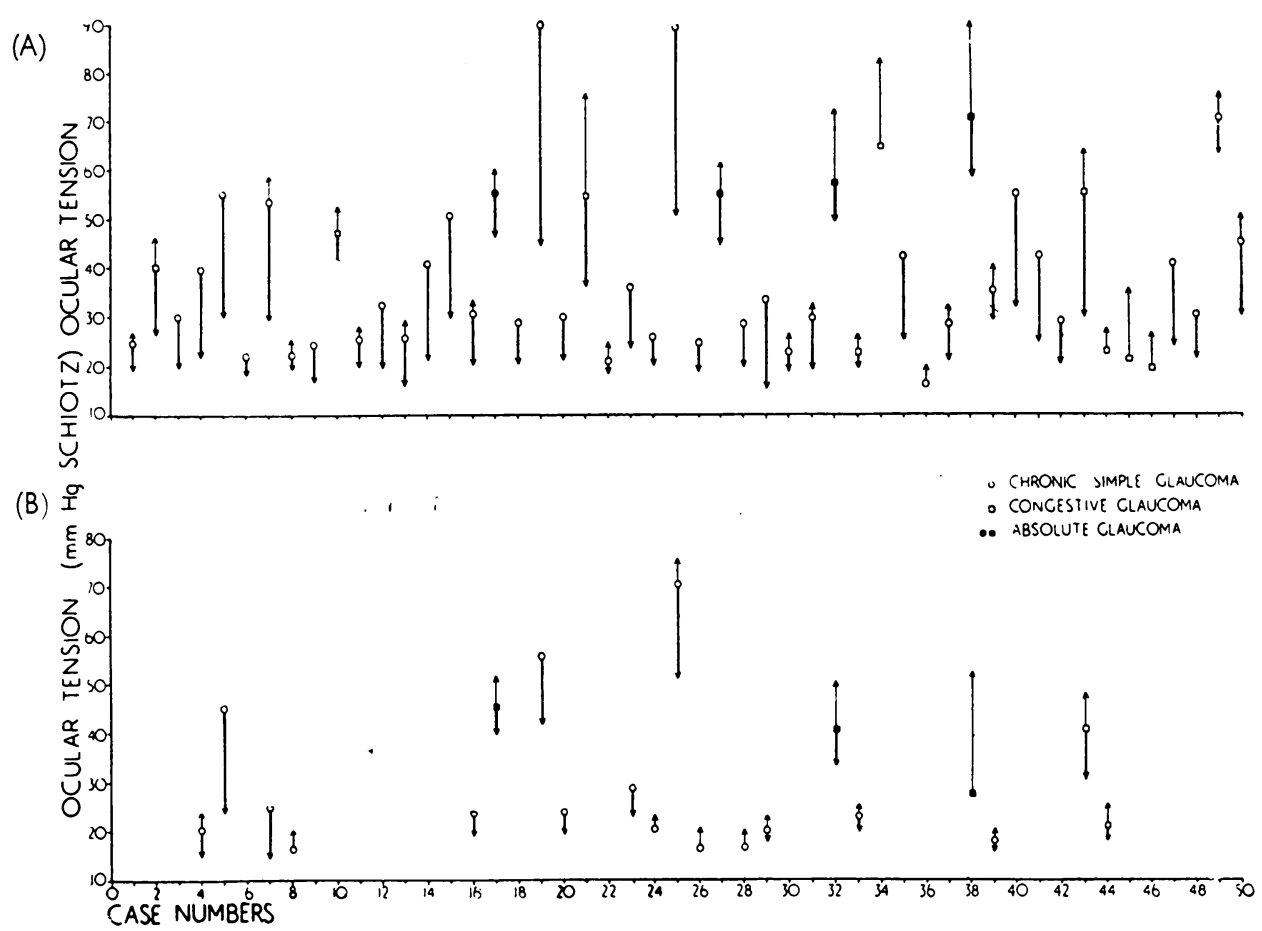

FIG. 1.-Amyl nitrite test in fifty patients with glaucoma.

(A) In basic conditions.

(B) Under cholinergic drugs. 
(3) After Blocking of Stellate Ganglion.-The variations in tension showed greater irregularity. They were mostly characterized by a fall reaching much lower values than the basal tension, and a short rise to a higher level (Fig. 2).

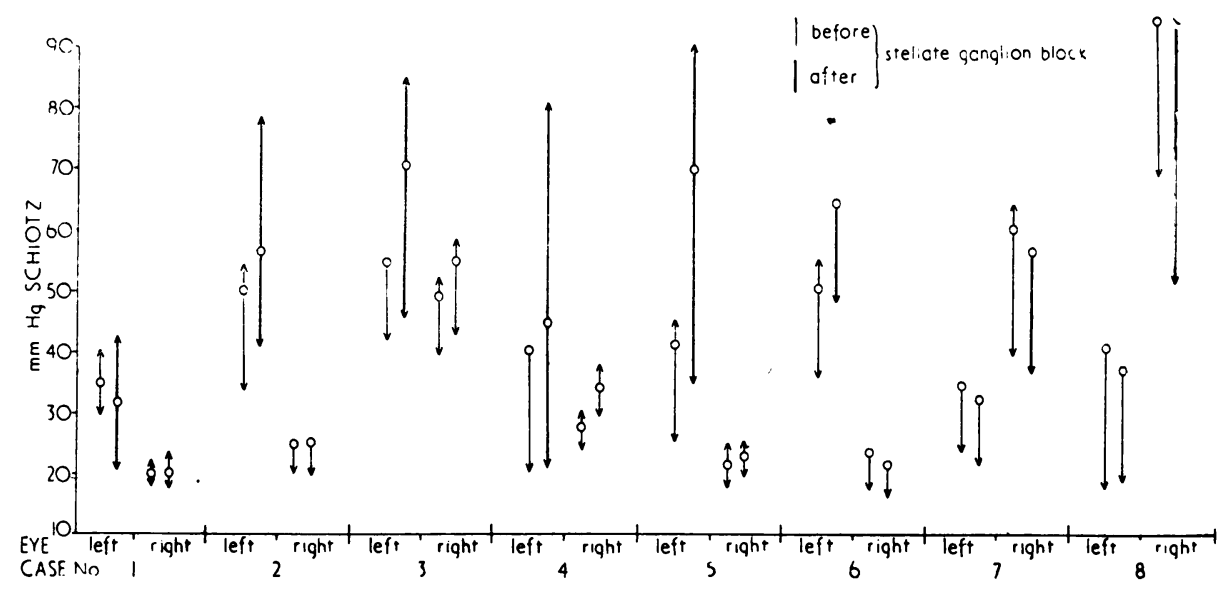

Fig. 2.-Amyl nitrite test in eight cases of primary glaucoma, before and after unilateral stellate ganglion block.

\section{Discussion}

As glaucomatous hypertension is determined by increased uveal capillary venous pressures, in consequence of a reduction of the circulatory bed of the small vessels, it is thought that the fall in basic intra-ocular pressure after the inspiration of amyl nitrite is caused by a diminution of the hydrostatic capillary venous pressures in the uvea following a widening of the capillary network. The rate of decrease, moreover, may be related to the widening capacity of the uveal capillary network; in cases where no fall occurs, impossibility of amplification must be deduced.

The response of intra-ocular pressure to amyl nitrite inspiration therefore acquires significance for the pathological evaluation of the symptom.

The insignificant fall in intra-ocular pressure registered in the two opposite pathological conditions, in cases with normal ocular tension and in hypertonic glaucomas during the absolute stage, would appear to prejudice the validity of the test. Though the result is the same in both conditions, i.e. no widening of the uveal capillary network occurs after the inspiration of amyl nitrite, the responsible pathological conditions are quite different. In glaucoma with normal tension, the uveal capillary network is presumably already at its maximum amplification through the dilatation of the lumina and the opening up of vascular reserve areas; in absolute glaucoma, on the contrary, the impossibility of any widening must be related to pathology of the uveal circulation due to an obliteration of the lumina and to a marked reduction of the capillary network. A confirmation of this conception in glaucoma with normal tension is given by the response of intra-ocular pressure to amyl nitrite after the instillation of cholinergic drugs. The 
vasodilatation elicited by these drugs, causing both dilatation of the lumina and the opening up of new uveal vascular areas, brings about the same condition as is spontaneously present in glaucoma with normal tension. This is the reason why the fall in intra-ocular pressure during the amyl nitrite test performed under the action of cholinergic drugs is small or absent.

Further investigations seem to be necessary for the interpretation of the behaviour of the ocular tension in cases of congestive glaucoma. Besides the absence of a fall after the inspiration of amyl nitrite-which might be understood on the basis discussed before-the ocular tension rose to a level higher than the initial values during the second phase. As a hypothesis, a defect in neurogenic control might be suggested, since the behaviour of intra-ocular pressure resembles that registered after sympathetic block.

\section{Summary}

The study of the amyl nitrite test in primary glaucoma performed in basic conditions, under the action of cholinergic drugs, and after unilateral blocking of the stellate ganglion, led to results which accord in general with the theory of a vascular pathogenesis of ocular hypertension in this disease.

It follows that the response to the amyl nitrite test may provide useful evidence for the clinical evaluation of the severity of this fundamental symptom in primary glaucoma.

\section{REFERENCES}

Ascher, K. W. (1949). Arch. Ophthal., Chicago, 42, 66.

Bailliart, P., and Bollack, J. (1921). Bull. Soc. franc. Ophtal., 34, 472.

Cristini, G. (1947). Ann. Oculist., Paris, 180, 530.

(1949). British Journal of Ophthalmology, 33, 228.

(1951a). "XVI Conc. Ophthal. Britannia Acta, 1950", vol. 2, p. 865. London. (1951b). British Journal of Ophthalmology, 35, 11.

Duke-Elder, S. (1952). Amer. J. Ophthal., 35, 1.

Goldmann, H. (1948). Ophthalmologica, Basel, 116, 195.

Greaves, D. P., and Perkins, E. S. (1952). British Journal of Ophthalmology, 36, 258.

LEYDHECKER, W. (1952). Klin. Mbl. Augenheilk., 121, 174.

Smith, Priestley $(1879,1891)$. "Glaucoma ". Churchill, London.

Thomassen, T. L. (1948). Trans. ophthal. Soc. U.K., 68, 75.

Urrets Zavalia, A. H. (1952). Ann. Oculist.. Paris, 185, 701. 